\title{
Colistin stimulates the activity of neutrophil elastase and Pseudomonas aeruginosa elastase
}

\author{
A. Jones, H. Elphick, E. Pettitt, M.L. Everard, G.S. Evans
}

Colistin stimulates the activity of neutrophil elastase and Pseudomonas aeruginosa elastase. A. Jones, H. Elphick, E. Pettitt, M.L. Everard, G.S. Evans. (C)ERS Journals Ltd 2002.

ABSTRACT: Nonantimicrobial effects of antibiotics may contribute to their activity in the treatment of infective airway disease. The aim of this study was to identify antibiotics used for the treatment of infection in cystic fibrosis that may alter the activity of human neutrophil elastase (HNE) and Pseudomonas aeruginosa elastase (PE).

The effect of antibiotics on the activity of purified HNE and PE, and HNE in sputum was assessed using colourimetric and fluorescent substrate assays by kinetic measurements, and by examining the interaction of HNE with inhibitors.

Ceftazidime, tobramycin, and gentamycin slightly inhibited purified HNE activity whereas erythromycin and colistin significantly stimulated purified HNE and PE (395 and $557 \%$, respectively). However, only colistin increased HNE activity in sputum $(+102 \%)$ and was therefore studied in more detail. This increase in activity was not due an interference with the specific inhibition of HNE by $\alpha_{1}$-antitrypsin but colistin was found to reverse the inhibitory effects of small molecular weight molecules like heparin.

Colistin increases the activity of human neutrophil elastase and Pseudomonas aeruginosa elastase, two proteases that contribute to the pathogenesis of cystic fibrosis airway disease.

Eur Respir J 2002; 19: 1136-1141.
Child Health-Division of Clinical Sciences, Sheffield Children's Hospital Trust, Sheffield, UK.

Correspondence: G.S. Evans, Child Health-Division of Clinical Sciences, Sheffield Children's Hospital Trust, Western Bank, Sheffield, S10 2TH, UK.

Fax: 441142755364

E-mail: g.s.evans@sheffield.ac.uk

Keywords: Colistin neutrophil elastase proteolysis

Pseudomonas

Received: March 262001

Accepted after revision January 18 2002

This study was partly funded by the Sheffield Hospital Children's Fund.
Antibiotics used to treat pulmonary infection represent one of the cornerstones of therapy for patients with cystic fibrosis (CF). A variety of antibiotics are used to treat endobronchial infection with the aim of delaying the progressive pulmonary damaging characteristics of this disease. Much of this damage is thought to be due to excessive proteases derived from neutrophils recruited into the airway in response to infectious organisms such as Staphylococcus aureus and Pseudomonas aeruginosa [1]. The latter organism also produces a thermolysin like elastase that can directly cause tissue damage as well as inactivating host antiproteinases such as $\alpha_{1}$-antitrypsin $\left(\alpha_{1}\right.$-AT) [2]. Aggressive antibiotic therapy combined with physiotherapy can effectively treat infective exacerbations and may also eradicate, or delay, colonization by pathogens such as Pseudomonas [3, 4].

Recent studies have suggested that antibiotics such as macrolides and aminoglycosides may have beneficial nonantimicrobial actions and that these may contribute to the clinical benefits observed when using these agents [5-7]. Macrolides appear to have potentially beneficial effects on cytokine production [8], neutrophil chemotaxis [9], inhibition of proteases $[10,11]$ and inhibition of Pseudomonas alginate biofilm synthesis [12]. It has also been reported that aminoglycosides, such as tobramycin and gentamycin, may have beneficial nonantimicrobial effects upon $P$. aeruginosa elastase (PE) [7].
The aim of the present study was to investigate whether other antibiotics commonly administered to $\mathrm{CF}$ patients change the activity of $\mathrm{HNE}$ and PE. The interactions of colistin with HNE were explored in detail, as this antibiotic was found to stimulate the protease in its purified form and in CF sputum. The mechanisms that led to increased protease activity were investigated with respect to direct effects upon HNE as well interactions of the enzyme with protease inhibitors.

\section{Materials and methods}

\section{Patient samples}

Sputum samples were collected with ethical approval from $10 \mathrm{CF}$ adolescent and adult patients (six males, mean age 15.6 yrs, range 9-22 yrs) during routine physiotherapy. The diagnosis of $\mathrm{CF}$ in all of the patients was confirmed by a positive sweat test. All of the patients were chronically colonized with $P$. aeruginosa (one patient was also confirmed with a Burkholdera cepacia infection) and the samples were taken during an acute exacerbation (average forced expiratory volume in one second $47 \%$ of normal with a range of $21-70 \%$ ). Immediately upon collection the samples were centrifuged using a Beckman J2-21 centrifuge (Beckman Coulter Division, Palo Alto, CA, 
USA) at $4{ }^{\circ} \mathrm{C}$ and $35,000 \times g$ for $150 \mathrm{~min}$ to clarify the sample and to remove cell debris and large deoxyribonucleic acid (DNA) fragments.

\section{Elastase colourimetric activity assay}

The activity of HNE (Elastin preoducts Inc., Owens ville, MO, USA) was measured with a specific peptide substrate methoxysuccinyl-ala-ala-pro-val-4-nitroanilide (MSAAP) (Sigma Chemicals, Poole, UK) [13], and purified HNE or sample was diluted in an assay buffer (0.1 M hydroxyethyl piperazine ethane sulphonic acid (HEPES), $0.5 \mathrm{M} \mathrm{NaCl}, 0.02 \mathrm{M} \mathrm{CaCl}_{2}, \mathrm{pH} 7.5$ ) [14]. To investigate the effects of antibiotics or HNE inhibitors, HNE and the test agent were added together for $10 \mathrm{~min}$ (at $20^{\circ} \mathrm{C}$ ) followed by MSAAP $\left(0.6 \mathrm{mg} \cdot \mathrm{mL}^{-1}\right)$. Samples were measured in triplicate and each experiment was repeated at least twice. The substrate and enzyme mixture was incubated for $\leqslant 2 \mathrm{~h}$ (at $20^{\circ} \mathrm{C}$ ) and measured at $410 \mathrm{nM}$ absorbence on a Titretek Multiskan MCC/340 colourimetric platereader (ICN Flow Laboratories). A standard curve of purified HNE (range 2-0.02 $\mathrm{U} \cdot \mathrm{mL}^{-1}$ ) was included within the assay.

\section{Effect of antibiotics on protease activity}

To investigate whether antibiotics stimulated or inhibited the activity of HNE, purified HNE $\left(2 \mathrm{U} \cdot \mathrm{mL}^{-1}\right)$ and sputum samples were mixed with a $0.5-0.05 \mathrm{mM}$ range of the antibiotics gentamycin (Baxter, Thetford, UK), gentamycin (+ethylenediamine tetraacetic acid), ceftadizime (Eli Lilly, Hampshire, UK), tobramycin (Eli Lilly), erythromycin (Abbott Laboratories, Kent, UK) and colistin. The effect of these antibiotics was measured after a 10-min pre-incubation followed by addition of the MSAAP substrate, and compared to the protease activity without the antibiotic. For those antibiotics available in saline or buffer, a similar volume of this diluent without the antibiotic was added to other wells to establish whether there were other interfering substances affecting protease activity. Colistin was also investigated further to examine its dose effects $(3.9-500 \mu \mathrm{M})$ upon purified $\mathrm{HNE}\left(2 \mathrm{U} \cdot \mathrm{mL}^{-1}\right)$ and HNE activity within sputum. Similar comparison was made of the dose-dependent effects of the detergent Brij-35 (3.9-500 $\mu \mathrm{M})$ (Sigma Chemicals) and Polymyxin B (Sigma Chemicals). Colistin and Brij-35 were also added $(50$ and $500 \mu \mathrm{M})$ to $10 \mathrm{CF}$ patient sputum samples to compare their effects upon the activity of HNE.

\section{Fluorescence quench assays}

To establish whether changes to HNE activity would also affect the degradation of macromolecular substrates, fluorescent-labelled placental type-IV collagen, gelatin, and Biodipy FL casein (Molecular Probes Inc, Eugene, Oregon, USA) were used. Purified HNE $\left(0.5 \mathrm{U} \cdot \mathrm{mL}^{-1}\right)$ or sputum, were incubated with colistin $(62.5-500 \mu \mathrm{M})$ diluted in assay buffer $(0.1 \mathrm{M}$
HEPES, 0.5 M NaCl, 0.02 $\mathrm{M} \mathrm{CaCl}_{2}, \mathrm{pH} 7.5$ ). After $10 \mathrm{~min}$ pre-incubation, the samples were added to the fluorescent substrates at $50 \mu \mathrm{g} \cdot \mathrm{mL}^{-1}$ (gelatin and type IV collagen) and $5 \mu \mathrm{g} \cdot \mathrm{mL}^{-1}$ (casein). The samples were mixed, incubated at $37^{\circ} \mathrm{C}$ in a humidified incubator for $24 \mathrm{~h}$ and the increase in fluorescence measured at an excitation wavelength of $485 \mathrm{nM}$ and emission of $530 \mathrm{nM}$ on a Denley Fluorescent platereader (Denley Scientific, Slough, Berkshire, UK). Changes in substrate degradation were measured against the samples without antibiotic (or detergent) but with an equivalent volume of buffer. Controls included the substrate and antibiotic combination without the sample, and the buffer and substrate but no antibiotic. Background autofluorescence of the antibiotic or buffer components was found to be very low but were subtracted from the sample readings. As each substrate yielded different levels of fluorescence, the results were standardized as the fold-increase in units of fluorescence over $24 \mathrm{~h}$ of incubation for the controls and samples with $500 \mu \mathrm{M}$ colistin.

\section{Effect of elastase inhibitors with and without colistin}

To determine whether colistin disrupted the inhibition of HNE by low molecular weight and specific antagonists, competition experiments were performed. The effect of $\alpha_{1}$-AT $(37.9 \mathrm{nM}-7 \mu \mathrm{M})$ [14], $N$-Methoxysuccinyl-ala-ala-pro-val-chloromethyl ketone (MSAAPket; Sigma Chemicals) (5 nM$50 \mu \mathrm{M})$ [15], and heparin $\left(15.6 \mathrm{ng}-1 \mu \mathrm{g} \cdot \mathrm{mL}^{-1}\right)$ [16] were tested on $4 \mathrm{U} \cdot \mathrm{mL}^{-1}$ of purified $\mathrm{HNE}$ and a $\mathrm{CF}$ sputum sample. $\alpha_{1}$-AT, MSAAPket and heparin were then used at the effective inhibitory dose at $50 \%$ (ID50) and 90\% (ID90) level concentrations and preincubated with different concentrations of antibiotic for $10 \mathrm{~min}$, followed by addition of purified HNE and the substrate. Changes in optical density (OD) at $410 \mathrm{nM}$ were recorded kinetically at 2-min intervals for $30 \mathrm{~min}\left(\right.$ at $\left.20^{\circ} \mathrm{C}\right)$. For each condition the increase in OD was plotted and linear regression was used to fit the best line. Controls included the enzyme and inhibitor but no antibiotic, the enzyme and antibiotic, and the enzyme and assay buffer alone. The effectiveness of these inhibitors upon HNE activity in sputum samples in the presence of the antibiotic was also determined at a single time point $(30 \mathrm{~min})$.

\section{Kinetic assay}

The effects of colistin on the kinetics of HNEsubstrate interaction was measured in terms of maximum initial reaction velocity $(V \max )$ and $\mathrm{Km}$ (the substrate concentration at half $V \max$ ). These values were determined kinetically with increasing MSAAP substrate $\left(0-2 \mathrm{mg} \cdot \mathrm{mL}^{-1}\right)$ concentration in the presence of $500 \mu \mathrm{M}$ colistin and $2 \mathrm{U} \cdot \mathrm{mL}^{-1}$ of sputum $\mathrm{HNE}$ at $20^{\circ} \mathrm{C}$. The increases in OD were measured at 2-min intervals for $30 \mathrm{~min}$ at OD $410 \mathrm{nM}$ and the values of $V \max$ and $\mathrm{Km}$ were determined by MichaelisMenten calculation. 
Effect of antibiotics on the activity of Pseudomonas aeuruginosa elastase

To determine whether antibiotics modified the activity of another type of protease implicated in the pathogenesis of CF, $25 \mathrm{nM}$ PE was incubated with or without 50 and $500 \mu \mathrm{M}$ concentrations of erythromycin, colistin, tobramycin, gentamycin and ceftazidine. The enzyme and antibiotic were mixed first and incubated for $10 \mathrm{~min}$ and then added to the fluorescent substrate. Changes in the fluorescence of the substrate were recorded as previously described.

\section{Statistics}

Effects of antibiotics and detergents on purified or sputum HNE were analysed for significance by the Mann-Whitney U-test and analysis of variance and by the Kruskall-Wallis procedure. For the inhibitor studies best-fit lines were calculated by linear regression and differences between the gradient of each slope by the t-test.

\section{Results}

Effect of antibiotics on human neutrophil elastase activity

Using a peptide-based substrate assay, ceftazidime, tobramycin and gentamycin caused inhibition of HNE activity by $42 \%, 30 \%$ and $28 \%$, respectively (fig. 1 ). In contrast, erythromycin and colistin significantly stimulated HNE activity (by $395 \%$ and 557\%, respectively). This stimulation was not due to constituents of the buffers used to dissolve the antibiotics. However, colistin stored at $4{ }^{\circ} \mathrm{C}$ for $>7$ days gradually lost its capacity to stimulate HNE.

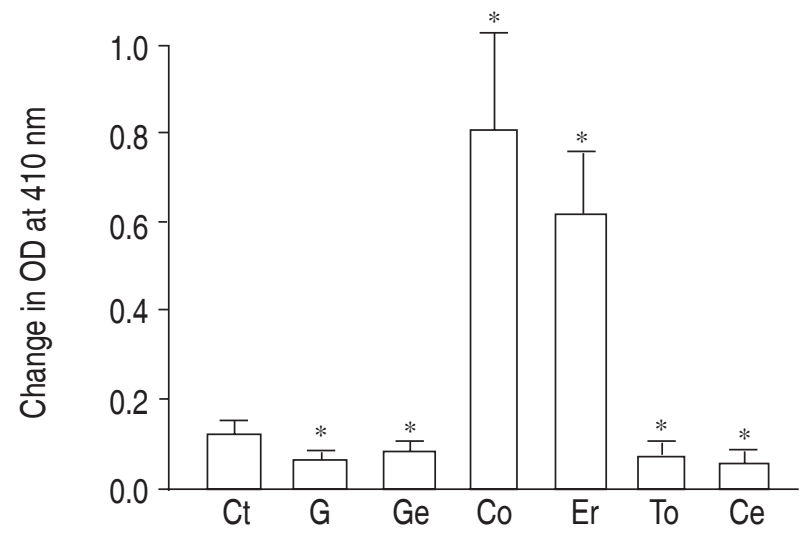

Fig. 1.-Measurement of the effect of the antibiotics gentamycin $(\mathrm{G})$, gentamycin+ethylenediamine tetraacetic acid $(\mathrm{Ge})$, colistin (Co), erythromycin (Er), tobramycin (To) and ceftazidime (Ce) $(500 \mu \mathrm{M})$ upon the activity of purified human neutrophil elastase (HNE) using the substrate $N$-(methoxysuccinyl)-ala-ala-pro-val chloromethyl ketone measured at an optical density of $410 \mathrm{nM}$. Control $(\mathrm{Ct})$ shows the activity of $\operatorname{HNE}\left(2 \mathrm{U} \cdot \mathrm{mL}^{-1}\right)$ in the presence of the buffer alone. Data are presented as the mean of three experiments with $95 \%$ confidence intervals. $*$ : $\mathrm{p}<0.05$ compared to control.
Colistin and erythromycin stimulate Pseudomonas aeruginosa elastase

Colistin and tobramycin, and to a greater extent erythromycin, stimulated PE when measured by degradation of Biodipy casein (fig. 2). The other antibiotics did not stimulate PE activity. The specificity of this activity was confirmed when phosphoramidon, an inhibitor of thermolysin-like enzymes, was added (fig. 2).

Colistin stimulates the activity of purified human neutrophil elastase and human neutrophil elastase in cystic fibrosis sputum

Of the antibiotics tested only colistin stimulated the activity of $\mathrm{HNE}$ in $\mathrm{CF}$ sputum. In the presence of increasing colistin concentration $(3.9-500 \mu \mathrm{M})$ both purified HNE and HNE in sputum were stimulated significantly in a dose-dependent manner (data not shown). For purified $\mathrm{HNE}, 3.9 \mu \mathrm{M}$ of colistin was sufficient to increase the activity significantly above the control $(162 \%, \mathrm{p}<0.01)$, and the maximal stimulation was observed at $250 \mu \mathrm{M}(877 \%, \mathrm{p}<0.001)$. For the $10 \mathrm{CF}$ sputum samples a $36 \%$ rise above background activity was observed at $32 \mu \mathrm{M}$ colistin $(\mathrm{n}=10, \mathrm{p}<0.05)$ and a $102 \%$ rise in activity at $500 \mu \mathrm{M}$ $(\mathrm{n}=10, \mathrm{p}<0.05)$. The stimulation obtained at $500 \mu \mathrm{M}$ colistin varied between $57-135 \%$.

Colistin increases the degradation of macromolecular substrates by elastase

The stimulatory effects of colistin were also found to affect the degradation of macromolecular substrates by HNE. HNE significantly increased degradation of gelatin $(36 \%)$, type IV collagen $(39 \%)$, and casein $(27 \%)$ in the presence of colistin (fig. 3, p<0.05).

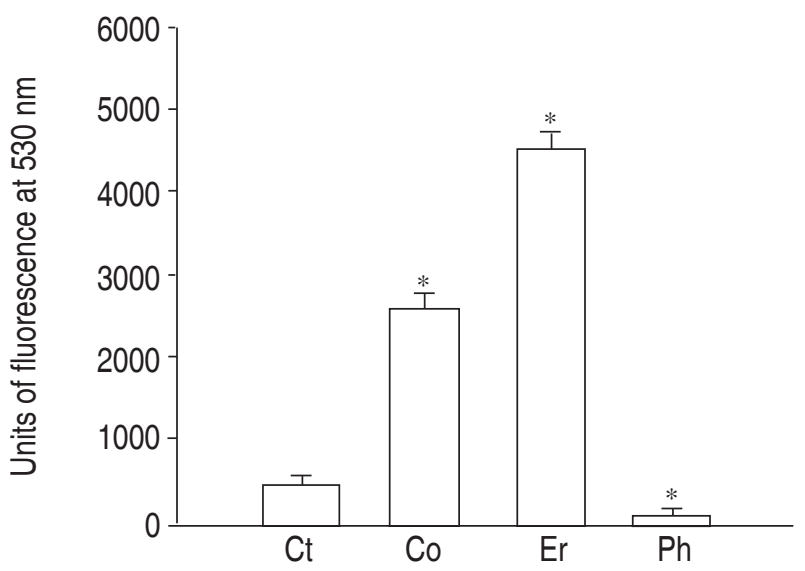

Fig. 2.-Measurement of the degradation of fluorescent quenched substrate Biodipy casein $\left(5 \mu \mathrm{g} \cdot \mathrm{mL}^{-1}\right)$ by $25 \mathrm{nM}$ Pseudomonas aeruginosa elastase $(\mathrm{Ct})$ at excitation $480 \mathrm{nM}$ and emission $530 \mathrm{nM}$ incubated in the presence of either $500 \mu \mathrm{M}$ colistin $(\mathrm{Co})$ or $500 \mu \mathrm{M}$ erythromycin (Er) and with $20 \mu \mathrm{g} \cdot \mathrm{mL}^{-1}$ phosphoramidon $(\mathrm{Ph})$ added. Data are presented as the mean of three experiments with $95 \%$ confidence intervals. $*$ : $p<0.05$ compared to control. 


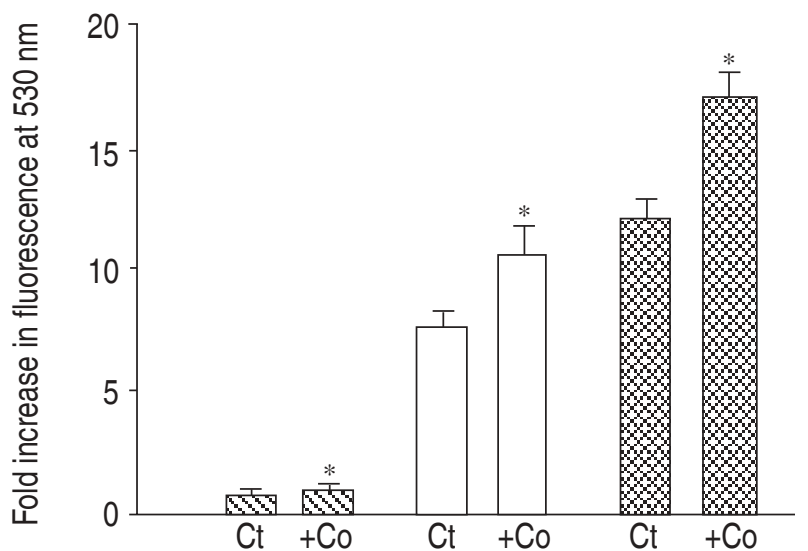

Fig. 3.-Measurement of the degradation of fluorescent quenched placental type-IV collagen $\left(50 \mu \mathrm{g} \cdot \mathrm{mL}^{-1}, \mathbf{2}\right)$, gelatin $\left(50 \mu \mathrm{g} \cdot \mathrm{mL}^{-1}\right.$, $\mathbb{\nabla})$ and Biodipy casein $\left(5 \mu \mathrm{g} \cdot \mathrm{mL}^{-1}, \square\right)$ by $0.8 \mathrm{U} \cdot \mathrm{mL}^{-1}$ of purified human neutrophil elastase (HNE) at excitation of $480 \mathrm{nM}$ and emission wavelengths of $530 \mathrm{nM}$. The $\mathrm{HNE}$ and substrates were incubated in the absence $(\mathrm{Ct})$ or presence $(+\mathrm{Co})$ of $500 \mu \mathrm{M}$ colistin. Data are presented as the mean of three experiments with $95 \%$ confidence intervals. * $: \mathrm{p}<0.05$ compared to control.

Colistin alone did not alter the fluorescence of these substrates (data not shown), and HNE preferentially degraded type IV collagen over gelatin and casein (fig. 3). However, the increases in HNE activity measured with the macromolecular substrates were not as great as those measured with the peptide-based substrates (fig. 1).

Detergents increase the activity of human neutrophil elastase

To demonstrate whether the detergent-like properties of colistin were responsible for increasing the activity of HNE, Brij-35 (3.9-500 $\mu \mathrm{M})$ was added to the buffer and was found to significantly increase maximal enzyme activity at $7.5 \mu \mathrm{M}(\mathrm{p}<0.001)$. A 33 -fold higher concentration $(250 \mu \mathrm{M})$ of colistin was required to stimulate the activity of $\mathrm{HNE}$ to the same level. Added at concentrations from 3.9-500 $\mu \mathrm{M}$ the related antibiotic Polymyxin B also stimulated HNE in an identical fashion to colistin (polymyxin E) (data not shown).

Colistin competes with low molecular weight inhibitors of elastase

The inhibitory concentration range of $\alpha_{1}-\mathrm{AT}$, MSAAPket and heparin, on purified HNE was determined and the IC50 and IC90 of each agent were added with or without the presence of $500 \mu \mathrm{M}$ colistin.

\section{$\alpha_{1}$-Antitrypsin}

At $900 \mathrm{nM}$ and $60 \mathrm{nM} \alpha_{1}$-AT completely inhibited HNE activity (data shown for $60 \mathrm{nM}$ dose in fig. 4a) compared to HNE alone. The addition of $500 \mu \mathrm{M}$
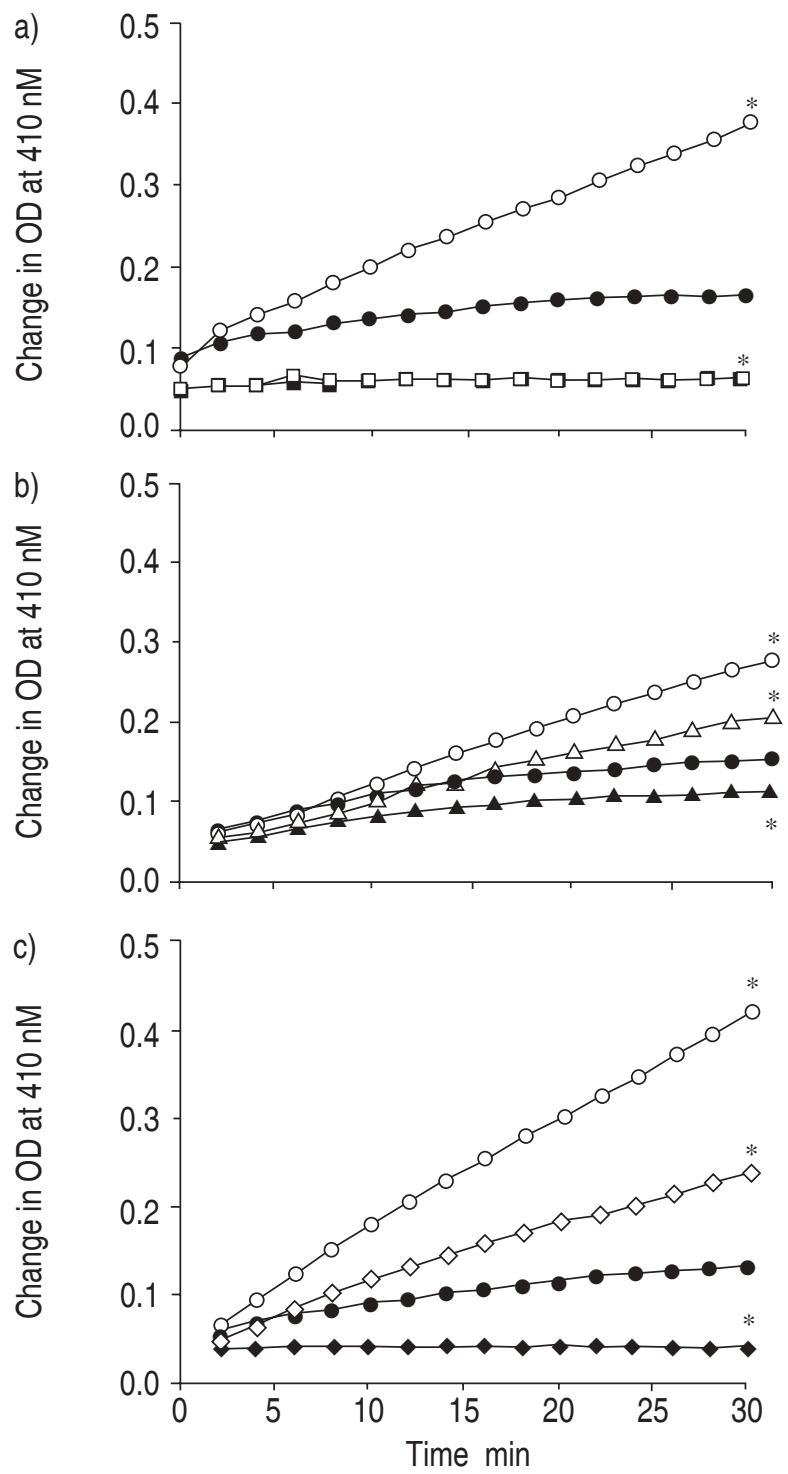

Fig. 4.-Measurement of the competition between a) $60 \mathrm{nM} \alpha_{1^{-}}$ antitrypsin (ם), b) $0.5 \mu \mathrm{M}$ N-(methoxysuccinyl)-ala-ala-pro-val chloromethyl ketone $(\boldsymbol{\Delta})$ or c) $15.6 \mathrm{ng} \cdot \mathrm{mL}^{-1}$ heparin $(\bullet)$, and human neutrophil elastase (HNE) (O) in the absence or presence of $500 \mu \mathrm{M}$ colistin $(\bigcirc)$. Data are presented as the mean of three. $*: \mathrm{p}<0.05$ in the gradient of the lines compared to control (HNE).

colistin alone significantly increased the activity of purified HNE (fig. 4a). Colistin was unable to reverse the inhibitory effects of $900 \mathrm{nM}$ (data not shown) and $60 \mathrm{nM}$ (fig. 4a) $\alpha_{1}$-AT on HNE activity.

\section{N-(Methoxysuccinyl)-ala-ala-pro-val-chloromethyl ketone}

MSAAPket inhibited HNE completely at $5 \mu \mathrm{M}$ and to a lesser extent at $0.5 \mu \mathrm{M}$ (fig. 4b). The addition of $500 \mu \mathrm{M}$ colistin alone significantly increased the activity of purified HNE (fig. 4b). Colistin was not able to reverse the inhibitory effects of $5 \mu \mathrm{M}$ MSAAPket but reversed the effects of $0.5 \mu \mathrm{M}$ 
MSAAPket (fig. 4b), although the activity was not as great when colistin alone was added to the enzyme.

\section{Heparin}

Heparin inhibited HNE activity completely at $1 \mu \mathrm{g} \cdot \mathrm{mL}^{-1}$ (data not shown) and $15.6 \mathrm{ng} \cdot \mathrm{mL}^{-1}$ (fig. 4c). The addition of $500 \mu \mathrm{M}$ colistin alone significantly increased the activity of purified HNE (fig. 4c). Colistin was able to partly reverse the effects of $1 \mu \mathrm{g} \cdot \mathrm{mL}^{-1}$ heparin (data not shown), and with $15.6 \mathrm{ng} \cdot \mathrm{mL}^{-1}$ heparin (fig. $4 \mathrm{c}$ ) the activity increased further above the control but not to the level observed when colistin alone was added to HNE.

\section{Colistin alters the kinetics of elastase activity}

Colistin increased the kinetics of HNE interaction with the MSAAP substrate. The values of $V \max$ and $\mathrm{Km}$ were 0.0043 rate of change in absorbance at $410 \mathrm{~nm} \cdot \min ^{-1}\left(\Delta \mathrm{A}_{410} \mathrm{~min}^{-1}\right)$ and $0.0946 \mathrm{M}$ for HNE alone and $0.0576 \Delta \mathrm{A}_{410} \mathrm{~min}^{-1}$ and $0.0776 \mathrm{M}$ for HNE and colistin, respectively.

\section{Discussion}

Nebulized colistin has been used for many years to treat patients with $\mathrm{CF}$ and in many countries it remains the most widely used nebulized antibiotic. It has been shown to be of value in eradicating $P$. aeruginosa in patients recently colonized with the organism and its use has been shown to reduce exacerbation in infected patients $[3,17]$. In light of the previously reported beneficial effects it was surprising to find that colistin increased the activity of HNE in purified preparations and sputum as well as that of PE.

These unexpected findings may be of clinical relevance to patients with chronic colonization with $P$. aeruginosa who are treated with long-term nebulized colistin. While published trials using nebulized colistin have involved treatment periods of $\leqslant 3$ months [17], many chronically colonized patients are treated with nebulized colistin for prolonged periods. In this setting it is possible that enhancement of HNE and PE activity will outweigh the positive antimicrobial effects of this agent. A recent study has provided in vivo evidence that prolonged administration of colistin is associated with airway damage in rats and dogs (Van Devanter, Chiron Corporation, Seattle, WA, USA, personal communication).

It was of interest that colistin stimulated both HNE and PE, which are serine- and thermolysin-like proteases, respectively. The effect upon HNE was seen in sputum samples at antibiotic concentrations comparable to nebulized doses assuming a heterogeneous delivery of $10-20 \%$ of the administered dose [18]. Unfortunately the lack of a specific substrate prevented the effect of colistin on PE activity within $\mathrm{CF}$ sputum being assessed. The mechanism by which colistin enhances HNE is unclear although the results of this study would suggest that its effect is mediated through the ability of the agent to interfere with the binding of certain inhibitor molecules. An identical dose-dependent stimulation of HNE was obtained with the related antibiotic polymyxin B. The polymyxins, including colistin, are small cyclic polypeptides with detergent-like properties with activity against Gram-negative bacteria [19]. It has previously been observered that the detergent Brij-35 is able to activate HNE [20] and it is likely that this effect is mediated through a similar mechanism.

In addition to antiproteases, such as $\alpha_{1}$-AT (or $\alpha_{1}$ antiprotease) and secretory leukoproteinase inhibitor, secretions from the inflamed airways contain molecules like heparin/heparan sulfate [16] and DNA [21], which also inhibit HNE activity. The negative charge of heparin binds to positively-charged groups on elastase and may account for its inhibition of activity [22]. In this present study a low concentration of heparin inhibited HNE but was unable to do so in the presence of $500 \mu \mathrm{M}$ colistin (fig. 4c). In contrast, $\alpha_{1}$-AT effectively inhibited HNE even when colistin was present. Colistin disrupted the ability of heparin to bind to HNE but was not able to disrupt the stable irreversible inhibitor-complexes formed with $\alpha_{1}$-AT. The ability of colistin to increase HNE activity in CF sputum suggests that $\alpha_{1}$-AT accounts for only a proportion of the HNE inhibitors present in $\mathrm{CF}$ sputum and that some of the enzyme may be bound to heparin like molecules, forming a pool susceptible to activation.

Why purified HNE showed a 10-fold increase in $V \max$ with colistin present was less clear. HNE prepared from sputum or purified neutrophils appeared pure on silver stained polyacrylamide gel electrophoresis gels (data not shown) but nevertheless may have contained small molecular contaminants like heparin. Many published studies where HNE activity has been measured have not reported the inclusion of detergents in the assay buffer. Therefore, the conditions for measuring HNE activity either purified or in respiratory secretions need to be defined carefully and Brij-35 containing buffers are essential to accurately measure the activity of HNE. It is likely that the reduced activity of colistin when added to CF sputum was due to absorption of the antibiotic into other charged molecules, since this fluid contains high concentrations of protein and DNA. Similarly, when inhibitors of HNE are added to sputum, they are less effective than when added to an equivalent activity of purified HNE (unpublished observations).

In conclusion, colistin increased the activity of both human neutrophil and Pseudomonas aeruginosa elastase, which are both believed to be important to the pathogenesis of the chronic lung disease characteristic of cystic fibrosis. The present study also showed that colistin increased the activity of human neutrophil elastase in cystic fibrosis sputum. These results suggest that further clinical studies are required. These studies should include the measurement of sputum elastase activity, and markers of tissue damage (e.g. urine levels of collagen and elastin fragments) pre and postcolistin inhalation. This will require the recruitment of a large number of patients since significant 
variation was observed in sputum markers between individuals.

\begin{abstract}
Acknowledgements. The authors would like to thank the Sheffield Hospital Children's Fund for financial support and K. Morihara (University of East Asia Yamaguchi 750, Japan) for the purified Pseudomonas aeruginosa elastase.
\end{abstract}

\section{References}

1. Suter S, Schaad UB, Roux L, Nydegger UE, Waldvogel FA. Granulocyte neutral proteases and pseudomonas elastase as possible causes of airway damage in patients with cystic fibrosis. J Inf Dis 1984; 149: 523-531.

2. Morihara K, Tzuzuki H, Oda K. Protease and elastase of Pseudomonas aeruginosa. inactivation of human plasma $\alpha 1$-proteinase inhibitor. Infect Immun 1979; 24: 188-193.

3. Littlewood JM, Smye SW, Cunliffe H. Aerosol antibiotic treatment in cystic fibrosis. Arch Dis Child 1993; 68: 788-792.

4. Frederiksen B, Koch C, Hølby N. Antibiotic treatment of initial colonization of Pseudomonas aeruginosa postpones chronic infection and prevents deterioration of pulmonary function in cystic fibrosis. Ped Pulmonol 1997; 23: 330-335.

5. Everard ML, Sly PD, Brennan S, Ryan G. Macrolide antibiotics in diffuse panbronchiolitis and in cystic fibrosis. Eur Respir J 1997; 10: 2926.

6. Jaffe A, Francis J, Rosenthal M, Bush A. Long-term azithromycin may improve lung function in children with cystic fibrosis. Lancet 1998; 381: 20.

7. O'Keeffe PT, Stewart GA, Sly PD. Antibiotic inhibition of Pseudomonas proteases. Am Rev Respir Dis 1993; 147: A29.

8. Abe $\mathrm{S}, \mathrm{Nakamura} \mathrm{H}$, Inoue $\mathrm{S}$, et al. Interleukin 8 gene repression by clarithromycin is mediated by the activator protein-1 binding site in human bronchial epithelial cells. Am J Respir Cell Mol Biol 2000; 22: 51-60.

9. Oda H, Kadota J, Kohno S, Hara K. Erythromycin inhibits neutrophil chemotaxis in bronchoalveoli of diffuse panbronchiolitis. Chest 1994; 106: 1116-1123.

10. Milwidsky A, Finchi-Yeheskel, Mayer M. Direct inhibition of proteases and cervical plasminogen activator by antibiotics. Am J Obst Gynecol 1992; 166: 606-612.

11. Sakata K, Yajima H, Tanaka K, et al. Erythromycin inhibits the production of elastases by Pseudomonas aeruginosa without affecting its proliferation in vitro. Am Rev Respir Dis 1993; 148: 1061-1065.

12. Yasuda H, Ajiki Y, Koga T, Kawada H, Yokota T. Interaction between biofilms formed by Pseudomonas aeruginosa and clarithromycin. Antimicrob Agents Chemother 1993; 37: 1749-1755.

13. Nakajima K, Powers JC, Ashe BM, Zimmerman M. Mapping the extended substrate binding site of cathepsin $G$ and human leukocyte elastase. Studies with peptide substrates related to the alpha 1-protease inhibitor reactive site. J Biol Chem 1979; 254: 40274032.

14. Cook L, Burdon JG, Brenton S, Knight KR, Janus ED. Kinetic characterization of $\alpha-1$ antitrypsin $F$ as an inhibitor of human neutrophil elastase. Comm Pathol 1997; 29: 331-333.

15. Stein RL, Trainor DA. Mechanism of inactivation of human leukocyte elastase by a chloromethyl ketone: kinetic and solvent isotope effect studies. Biochem 1986; 25: 5414-5419.

16. Volpi N. Inhibition of human leukocyte elastase activity by heparins: influence of charge density. Biochim Biophys Acta 1996; 1290: 299-307.

17. Frederiksen D, Koch C, Høiby N. Antibiotic treatment of initial colonization with Pseudomonas aeruginosa postpones chronic infection and prevents deterioration of pulmonary function in cystic fibrosis. Pediatr Pulmonol 1997; 23: 330-335.

18. Gagnadoux F, Diot P, Marchand S, et al. Pulmonary deposition of colistin aerosols in cystic fibrosis. Comparison of an ultrasonic nebulizer and a pneumatic nebulizer. Rev Mal Respir 1996; 13: 55-60.

19. Goodwin NJ. Colistin and sodium colistimethate. Med Clin N America 1970; 54: 1267-1276.

20. Cook L, Tema B. Deleterious effect of Brij-35 on alkyl 2-pyrones and other hydrophobic inhibitors of human sputum and leukocyte elastase. Biochem Int 1988; 17: 637-646.

21. Berlorgey D, Bieth JG. DNA binds neutrophil elastase and mucus proteinase inhibitor and impairs their functional activity. FEBS Letts 1995; 361: 265-268.

22. Hornebeck W, Lafuma C, Robert L, Moczar M, Moczar E. Heparin and its derivatives modulate serine proteinases (SRPS) serine proteinase inhibitor (SERPINS) balance. Pathol Res Pract 1994; 190: 895902. 